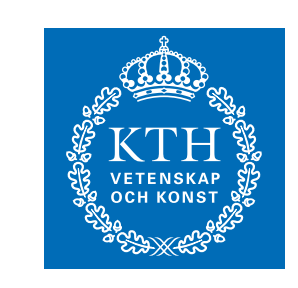

КTH Electrical Engineering

\title{
Approximating the Capacity of Wireless Multiple Unicast Networks by Discrete Superposition Model
}

(C)2012 IEEE. Personal use of this material is permitted. However, permission to reprint/republish this material for advertising or promotional purposes or for creating new collective works for resale or redistribution to servers or lists, or to reuse any copyrighted component of this work in other works must be obtained from the IEEE.

NICOLAS SCHRAMMAR AND MIKAEL SKOGLUND

Stockholm 2012

Communication Theory Lab

School of Electrical Engineering

KTH Royal Institute of Technology 


\title{
Approximating the Capacity of Wireless Multiple Unicast Networks by Discrete Superposition Model
}

\author{
Nicolas Schrammar and Mikael Skoglund \\ School of Electrical Engineering and ACCESS Linnaeus Centre \\ KTH Royal Institute of Technology, Stockholm, Sweden \\ Email: \{nisc, skoglund\}@kth.se
}

\begin{abstract}
The discrete superposition model (DSM) is intended to approximate the capacity region of AWGN networks. Finding the capacity region in the DSM is simpler due to its discrete and deterministic properties. For unicast in relay networks and for the multi-user interference channel it has been shown that the capacity regions of the DSM and of the AWGN model are within a constant gap. We extend this result to multiple unicast in networks consisting of broadcast and multiple-access channels by using a recent result on polymatroidal networks. We show that the capacity regions of the two models are within a constant additive gap and a constant multiplicative gap.
\end{abstract}

\section{INTRODUCTION}

The extension of Shannon's results on the capacity of pointto-point channels to large communication networks appears to be a difficult task. The capacity regions of some singlehop channels such as the multiple-access channel (MAC) and the degraded broadcast channel (BC) have been found. However, there are simple examples such as the three-node relay channel, where capacity regions are unknown, in spite of considerable research efforts over many years [1].

Recently, there has been some progress in approximating the capacity regions of more complicated channels. Important examples include the unicast relay network [2] and the twouser interference channel [3] in the additive white Gaussian noise (AWGN) model. For those examples, the capacity (region) has been found within a constant additive gap. Such an approximation is useful in the high signal-to-noise ratio (SNR) regime, where the cap becomes negligible compared to the capacity.

Deterministic channel models [2] provide a systematic way of finding constant-gap approximations, if the model is chose such that its capacity approximates the corresponding AWGN capacity. This is because finding the capacity in deterministic models can be much easier than finding the AWGN capacity. Models that approximate the AWGN model in this way include the truncated deterministic model [2] and the discrete superposition model (DSM) [4]. Both models approximate the AWGN capacity of unicast relay networks, that is, networks with one source, one destination and arbitrary many relays with arbitrary interconnection [2], [4]. Furthermore, the DSM approximates the capacity region of $K$-user interference channels [4].

This work was supported by the Swedish research council (VR).

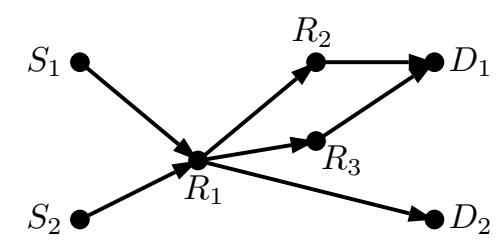

Fig. 1. Multiple-unicast network with $k=2$ source-destinations pairs consisting of $\mathrm{BC}$ and MAC components.

In this paper we prove that the DSM approximates the AWGN capacity region of a class of multiple-unicast networks. In those networks there exist $k$ sources that transmit information to one of $k$ destinations each. An arbitrary number of relays assist in the transmission. We consider multiple-unicast networks that consist of disjoint BC and MAC components, that is, every link in the network belongs to either a $\mathrm{BC}$ component or a MAC component. An example for such a network with $k=2$ source-destination pairs is depicted in Fig. 1. This requirement might appear restrictive. Note, however, that any network can be operated in a frequencydivision (or time-division) scheme, where links operating at the same frequency are selected such that they form a network consisting of $\mathrm{BC}$ and MAC components [5].

To prove this correspondence between AWGN model and DSM, we use a property of polymatroidal networks [5]. In such networks, there is a connection between the minimumcut region and the maximum-flow region for multiple-unicast, which resembles the famous min-cut-max-flow theorem [6]. We then show that the AWGN network and the DSM network can be approximated by the same polymatroidal network, both in terms of minimum cut and maximum flow. The reason for considering networks consisting of $\mathrm{BC}$ and MAC components is that a cut in those networks separates into cuts in the components. This offers a crucial simplification in evaluating the min-cut region.

The remainder of this paper is organized as follows: We review the concept of polymatroidal networks and the two channel models in Sec. II. In Sec. III we state and prove our result. Sec. IV discusses the result and concludes the paper. 


\section{System Model}

\section{A. Graph Representation and Polymatroids}

Consider a directed graph $G=(V, E)$ consisting of a set $V$ of nodes and a set $E$ of directed links $(u, v)$ from node $u \in V$ to node $v \in V$. Define $\delta^{\text {in }}(v)$ as the set of links in to node $v$, i.e.

$$
\delta^{\text {in }}(v)=\{(x, y) \in E \mid y=v\} .
$$

Similarly, define $\delta^{\text {out }}(v)$ as the set of links out of node $v$, i.e.

$$
\delta^{\text {out }}(v)=\{(x, y) \in E \mid x=v\} .
$$

We call the number of links in to and out of node $v$ the in-degree $\operatorname{deg}^{\text {in }}(v)=\left|\delta^{\text {in }}(v)\right|$ and out-degree $\operatorname{deg}^{\text {out }}(v)=$ $\left|\delta^{\text {out }}(v)\right|$, respectively.

A node $S$ with in-degree $\operatorname{deg}^{\mathrm{in}}(S)=0$ is called a source; a node $D$ with out-degree $\operatorname{deg}^{\text {out }}(D)=0$ is called a destination. Our network, represented by the graph $G$, contains $k$ sourcedestination pairs $\left(S_{1}, D_{1}\right), \ldots,\left(S_{k}, D_{k}\right)$. Each source $S_{i}$ seeks to transmit a message $w_{i}$ to its destination $D_{i}$ at a rate $R_{i}$ by using block codes of length $n$. This mode of communication is termed multiple-unicast. A rate vector $\boldsymbol{R}=\left(R_{1}, \ldots, R_{k}\right)$ is achievable if we can find codes with rates $R_{i}$ and block length $n$ for any desired error probability $P_{e}>0$ of the codes. The capacity region $\mathcal{C}$ is the closure of all achievable rate vectors.

In this paper we consider networks that consist of $\mathrm{BC}$ and MAC components. A BC component of degree $d$ consists of a node $u$ with out-degree $\operatorname{deg}^{\text {out }}(u)=d$, that is connected to $d$ nodes $v_{i}$ with in-degree $\operatorname{deg}^{\text {in }}\left(v_{i}\right)=1$. Similarly, a MAC component consists of $d$ nodes $u_{i}$ with out-degree $\operatorname{deg}^{\text {out }}\left(u_{i}\right)=1$ and a node $v$ with in-degree $\operatorname{deg}^{\text {in }}(v)=d$. A network $G$ consists of $\mathrm{BC}$ and MAC components, if for each link $(u, v) \in E$ from $u$ to $v$ either $\operatorname{deg}^{\text {out }}(u)=1$ or $\operatorname{deg}^{\text {in }}(v)=1$ or both. Fig. 1 shows a network consisting of $\mathrm{BC}$ and MAC components.

In many communication networks, including wire-line networks, each link has a capacity which limits the rate of information sent over this link. In a wireless scenario, however, this might not be a suitable model, since multiple links entering or departing a node interact with each other. To capture this effect, we introduce additional constraints on the sum-rate sent over a set of links. If these constraints have a polymatroidal structure as described below, there exist useful connections between the maximum flow and the minimum cut in a network [5].

A set function $f: P(N) \rightarrow \mathbb{R}$ assigns a real value to any subset of the set $N$. It is submodular if $f(A)+f(B) \geq$ $f(A \cap B)+f(A \cup B)$ for all $A, B \subseteq N$. It is monotone if $f(A) \leq f(B)$ for all $A \subseteq B$. We refer to the region

$$
\left\{\left(x_{1}, \ldots, x_{|N|}\right) \mid \sum_{i \in S} x_{i} \leq f(S) \text { for all } S \subseteq N\right\},
$$

in $\mathbb{R}^{|N|}$ as polymatroid if $f$ is a monotone submodular function with $f(\emptyset)=0$. For simplicity, we will refer to the function $f$ itself as polymatroid.
In a polymatroidal network [5], the sum-rate of information in a subset $S \subseteq \delta^{\text {in }}(v)$ of links in to a node $v$ is constrained by the polymatroid $\rho^{\text {in }}(v)$. Similarly, the links out of a node are constrained by the polymatroid $\rho^{\text {out }}$. Finally, a bidirected polymatroidal network is formed from a directed polymatroidal network by adding for each link $(u, v) \in E$ the corresponding reverse link $(v, u)$ and assigning polymatroidal capacity constraint that correspond to the constraints of $(u, v)$.

\section{B. Maximum Flow and Minimum Cut}

A single-commodity flow from source $S$ to destination $D$ corresponds to an assignment of rates to the links in the network, such that information can be routed from $S$ to $D$ at rate $R$. The information can flow on multiple paths through the network, but the rate on every link in the network must not exceed its capacity constraints.

A multi-commodity flow in a multiple unicast network with $k$ source-destination pairs consists of a $k$ single-commodity flows with rates $R_{1}, \ldots, R_{k}$. The accumulated rate from the $k$ commodities must not exceed the capacity constraints on any link. The region of rate tuples $\left(R_{1}, \ldots, R_{k}\right)$ for which a multi-commodity flow exists is referred to as max-flow rate region $\mathcal{R}_{\text {flow }}$.

The cut-set bound in a multiple unicast network is

$$
\sum_{i: S_{i} \in \mathcal{S}, D_{i} \in \overline{\mathcal{S}}} R_{i} \leq I((X(\mathcal{S}) ; Y(\overline{\mathcal{S}}) \mid X(\overline{\mathcal{S}}))=\nu(\mathcal{S}),
$$

where $X(\mathcal{S})$ is the vector of signals transmitted by the nodes in $\mathcal{S}$, and $Y(\overline{\mathcal{S}})$ is the vector containing the signals received by nodes in $\mathcal{S}$. The bound holds for all separations of the nodes in $V$ into the sets $\mathcal{S} \subseteq V$ and $\overline{\mathcal{S}}=V \backslash \mathcal{S}$. It means that the sum of the rates of the separated source-destination pairs cannot be larger than the value $\nu(\mathcal{S})$ of the cut. In a network with only link-capacity constraints, $\nu(\mathcal{S})$ is just the sum of the capacities of the links from nodes in $\mathcal{S}$ to nodes in $\overline{\mathcal{S}}$. For a general network with polymatroidal constraints the value of a cut is more difficult to obtain as described in [5, Definition 1]. However, for networks consisting of BC and MAC components, the total value of the cut is just the sum of the values of the component cuts [5, Proof of Lemma 13].

The min-cut rate region $\mathcal{R}_{\text {cut }}$ is the region of rate tuples $\left(R_{1}, \ldots, R_{k}\right)$, for which (4) is fulfilled for all $\mathcal{S} \subseteq V$.

The following theorem is essential for proving our result:

Theorem 1. [5, Theorem 1]. For a bidirected polymatroidal network with $k$ source-destination pairs, the ratio between the max-flow region $\mathcal{R}_{\mathrm{ach}}^{\mathrm{poly}}$ and the min-cut region $\mathcal{R}_{\mathrm{cut}}^{\mathrm{poly}}$ is $\mathcal{O}(\log k)$.

\section{Channel Models}

We use two channel models to capture the interaction of the wireless medium: the AWGN model and the DSM. In the AWGN model the signal received at node $v$ is

$$
y_{v}=\sum_{u \in \delta^{\text {in }}(v)} h_{u v} x_{u}+z_{v},
$$


where $h_{u v}$ is the complex-valued gain of the channel from $u$ to $v, x_{u}$ is the complex-valued transmit signal at transmitter $u$, and $z_{v} \sim \mathcal{C N}(0,1)$ is zero-mean, complex Gaussian noise of unit variance. We normalize the transmit power $\mathrm{E}\left|x_{u}\right|^{2} \leq 1$, hence, the network is described by the set of channel gains.

We use the same set of channel gains to define the corresponding DSM as in [4]. The receive signal at node $v$ is

$$
y_{v}=\sum_{u \in \delta_{\text {in }}(v)}\left[\left[h_{u v}\right] x_{u}\right]
$$

where the quantization function [.] is defined as

$$
[a]=\operatorname{sgn}\left(a_{\operatorname{Re}}\right)\left\lfloor\left|a_{\operatorname{Re}}\right|\right\rfloor+j \operatorname{sgn}\left(a_{\operatorname{Im}}\right)\left\lfloor\left|a_{\operatorname{Im}}\right|\right\rfloor .
$$

It rounds the real part $a_{\mathrm{Re}}$ and the imaginary part $a_{\mathrm{Im}}$ of $a$ towards the closest integer with smaller absolute value. Furthermore, the channel inputs $x_{u}$ can take only discrete values from the set

$$
\left\{0, \frac{2^{-n}}{\sqrt{2}}, \ldots, \frac{1-2^{-n}}{\sqrt{2}}\right\}+j\left\{0, \frac{2^{-n}}{\sqrt{2}}, \ldots, \frac{1-2^{-n}}{\sqrt{2}}\right\}
$$

of equidistantly spaced complex values. The number $2^{2 n}$ of channel inputs depends on the maximum channel gain through

$$
n=\max _{(u, v) \in E}\left\lfloor\log \left(\max \left\{\left|h_{u v, \operatorname{Re}}\right|,\left|h_{u v, \operatorname{Im}}\right|\right\}\right)\right\rfloor .
$$

All logarithms in this paper are base 2 .

\section{MAIN RESULT}

In this section we prove the following relation between the AWGN capacity region $\mathcal{C}^{\mathrm{AWGN}}$ and the DSM capacity region $\mathcal{C}^{\text {DSM }}$. We provide some discussion and interpretation in the subsequent section.

Theorem 2. The capacity regions of the DSM $\mathcal{C}^{\mathrm{DSM}}$ and the AWGN model $\mathcal{C}^{\text {AWGN }}$ are within a constant additive gap and a constant multiplicative gap: If $\boldsymbol{R}^{\mathrm{DSM}}$ is an achievable rate vector in the DSM, there exists an achievable rate vector $\boldsymbol{R}^{\mathrm{AWGN}}$ in the AWGN model with

$$
R_{i}^{\mathrm{AWGN}} \geq \frac{R_{i}^{\mathrm{DSM}}-\alpha}{\mu}, \quad i=1,2, \ldots, k .
$$

Conversely, if $\boldsymbol{R}^{\mathrm{AWGN}}$ is an achievable rate vector in the $A W G N$ model, there exists an achievable rate vector $\boldsymbol{R}^{\mathrm{DSM}}$ in the DSM with

$$
R_{i}^{\mathrm{DSM}} \geq \frac{R_{i}^{\mathrm{AWGN}}-\alpha}{\mu}, \quad i=1,2, \ldots, k .
$$

The additive gap is at most $\alpha \leq 10 c_{\max } d_{\max } \log d_{\max }$, where $d_{\max }$ is the maximum degree of the $B C$ and MAC components, and $c_{\max }$ is the maximum number of $B C$ and MAC components included in a cut. The multiplicative gap is $\mu=\mathcal{O}(\log k)$.

Proof: Consider a graph $G=(E, V)$ and a set of channel gains $h_{u v}$ for every link $(u, v) \in V$. This set constitutes the AWGN model and the DSM of our network according to Section II-C. In order to prove the two relations

$$
\begin{aligned}
\mu \mathcal{R}_{\mathrm{ach}}^{\mathrm{DSM}}+\alpha \supseteq \mathcal{R}_{\mathrm{ach}}^{\mathrm{AWGN}}, \\
\mu \mathcal{R}_{\mathrm{ach}}^{\mathrm{AWGN}}+\alpha \supseteq \mathcal{R}_{\mathrm{ach}}^{\mathrm{DSM}},
\end{aligned}
$$

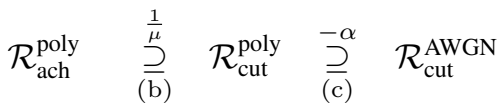

$$
\begin{aligned}
& \cap I(a) \quad \text { (d) } I U \\
& \mathcal{R}_{\text {ach }}^{\mathrm{DSM}} \quad \mathcal{R}_{\text {ach }}^{\mathrm{AWGN}} \\
& \mathrm{nI}(\mathrm{h}) \quad \text { (e)IU } \\
& \mathcal{R}_{\text {cut }}^{\text {DSM }} \underset{(\mathrm{g})}{\stackrel{+\alpha}{\subseteq}} \mathcal{R}_{\text {cut }}^{\text {poly }} \underset{(\mathrm{f})}{\stackrel{\mu}{\complement}} \mathcal{R}_{\text {ach }}^{\text {poly }}
\end{aligned}
$$

Fig. 2. Proof structure of Theorem 2

we define a third network with the following polynomial constraints:

$$
\begin{aligned}
\rho^{\text {in }}(v) & =\left(\max _{u \in \delta^{\text {in }}(v)} \log \left|h_{u v}\right|^{2}-7 d_{\text {max }} \log d_{\text {max }}\right)^{+}, \\
\rho^{\text {out }}(u) & =\left(\max _{v \in \delta_{\text {out }}(u)} \log \left|h_{u v}\right|^{2}-7 d_{\max } \log d_{\text {max }}\right)^{+},
\end{aligned}
$$

where $\rho^{\text {in }}(v)$ and $\rho^{\text {out }}(v)$ are polymatroids constraining the flow in to and out of node $v$, respectively. The max-flow region and the min-cut region of the polymatroidal network are denoted by $\mathcal{R}_{\text {ach }}^{\text {poly }}$ and $\mathcal{R}_{\text {cut }}^{\text {poly }}$, respectively. We use the polymatroidal network to make the connection between the max-flow and the min-cut region. We then show that maxflow and min-cut regions in the AWGN model and the DSM are also approximately polymatroidal, which yields the result. This approach is similar to the proof of [5, Theorem 5], where it is used to bound the gap between the min-cut region and the max-flow region of AWGN networks. The structure of the proof is depicted in Fig. 2.

We begin by considering the immediate steps. Construct a bidirected polymatroidal network by adding the link $(v, u)$ for each link $(u, v) \in V$. The link has the same channel gain $h_{v u}=h_{u v}$ due to the reciprocity of the wireless medium. Theorem 1 provides

$$
\mu \mathcal{R}_{\text {ach }}^{\text {poly }} \supseteq \mathcal{R}_{\text {cut }}^{\text {poly }},
$$

which yields steps (b) and (f). Also, steps (d) and (h) follow from the cut-set bound theorem [1]: The min-cut region is an outer bound to the max-flow region.

The remaining steps concern the connection between the polymatroidal network and the AWGN or DSM network. Consider an AWGN MAC with a set $V$ of transmitters. For every subset $\mathcal{S} \subseteq V$ we can achieve the following sumrate choosing the transmit signals $X_{i}, i \in \mathcal{S}$ Gaussian and independent of each other [1]:

$$
\begin{aligned}
\sum_{i \in \mathcal{S}} R_{i, \mathrm{MAC}}^{\mathrm{AWGN}} & =I\left(X_{\mathcal{S}} ; Y \mid X_{\overline{\mathcal{S}}}\right) \\
& =h\left(Y \mid X_{\overline{\mathcal{S}}}\right)-h\left(Y \mid X_{V}\right) \\
& =h\left(\sum_{i \in X_{\mathcal{S}}} h_{i} X_{i}+Z\right)-h(Z)
\end{aligned}
$$




$$
\begin{aligned}
& =\log \left(1+\mathrm{E}\left(\sum_{i \in X_{\mathcal{S}}} \sum_{j \in X_{\mathcal{S}}} h_{i} X_{i} h_{j}^{*} X_{j}^{*}\right)\right) \\
& =\log \left(1+\sum_{i \in X_{\mathcal{S}}}\left|h_{i}\right|^{2}\right) \\
& \geq \log \left(\max _{i \in X_{\mathcal{S}}}\left|h_{i}\right|^{2}\right),
\end{aligned}
$$

which is larger than the constraint $\rho^{\text {out }}$ in the polymatroidal network.

Consider the $\mathrm{BC}$ with the same set of channel gains $h_{i}$. If the transmitter had a power constraint of $\mathrm{E}|X|^{2} \leq|V|$, we could achieve the same rate region as in the corresponding MAC [5]. Hence, for $\mathrm{E}|X|^{2} \leq 1$ we can achieve

$$
\begin{aligned}
\sum_{i \in \mathcal{S}} R_{i, \mathrm{BC}}^{\mathrm{AWGN}} & =\log \left(\max _{i \in X_{\mathcal{S}}}\left|h_{i}\right|^{2}\right)-\log |V| \\
& \geq \log \left(\max _{i \in X_{\mathcal{S}}}\left|h_{i}\right|^{2}\right)-d_{\max }
\end{aligned}
$$

for every $\mathcal{S} \subseteq V$. This is larger than $\rho^{\text {out }}$. So on every BC and MAC component in the AWGN model we can achieve the same rate region as in the polymatroidal network. Therefore, we can also achieve the same rate region in the multipleunicast network by using decode-and-forward. This yields step (e).

Concerning step (c), we show that the value of any cut $\mathcal{S} \subseteq V$ in the AWGN model is at most $\alpha$ below the value of the same cut in the polymatroidal network. This yields $\mathcal{R}_{\text {cut }}^{\mathrm{AWGN}} \subseteq \mathcal{R}_{\text {cut }}^{\text {poly }}+\alpha$. Due to [5, Section 2.2] the cut-set bound (4) separates as

$$
I\left((X(\mathcal{S}) ; Y(\overline{\mathcal{S}}) \mid X(\overline{\mathcal{S}})) \leq \sum_{c} I((X(\mathcal{S}, c) ; Y(\overline{\mathcal{S}}, c) \mid X(\overline{\mathcal{S}}, c))\right.
$$

into cut-set bounds of independent component channels $c$. Furthermore, the relation holds with equality in the polymatroidal network [5]. In our network, the independent components are the $\mathrm{BC}$ and MAC components of the channel.

Concerning the cut-set bound on the MAC in the AWGN model, note that the common assumption of independent transmit signals $X_{i}$ does not apply, if the MAC is used as part of a larger network. For an arbitrary distribution of transmit signals, the maximum value of the cut is given by the capacity of the corresponding MISO channel with a total power constraint $|\mathcal{S}|$ :

$$
\begin{aligned}
I\left(\left(X_{\mathrm{MAC}}^{\mathrm{AWGN}}(\mathcal{S}) ; Y_{\mathrm{MAC}}^{\mathrm{AWGN}}\right)\right. & \leq \log \left(1+|\mathcal{S}| \sum_{i \in \mathcal{S}}\left|h_{i}\right|^{2}\right) \\
& \leq \log \left(2|\mathcal{S}|^{2} \max _{i \in \mathcal{S}}\left|h_{i}\right|^{2}\right) \\
& \leq \log \left(\max _{i \in \mathcal{S}}\left|h_{i}\right|^{2}\right)+2 d_{\max } \log d_{\max } \\
& \leq \rho^{\text {in }}+9 d_{\text {max }} \log d_{\text {max }} .
\end{aligned}
$$

Similarly, the maximum value of a cut $\mathcal{S}$ through a $\mathrm{BC}$ component equals the capacity of the corresponding SIMO channel, because the receivers can cooperate. This yields

$$
\begin{aligned}
I\left(\left(X_{\mathrm{BC}}^{\mathrm{AWGN}} ; Y_{\mathrm{BC}}^{\mathrm{AWGN}}(\overline{\mathcal{S}})\right)\right. & \leq \log \left(1+\sum_{i \in \mathcal{S}}\left|h_{i}\right|^{2}\right) \\
& \leq \rho^{\text {out }}+8 d_{\max } \log d_{\max }
\end{aligned}
$$

by following similar steps as above. Equations (20) and (21) yield

$$
\begin{aligned}
& I\left(\left(X^{\mathrm{AWGN}}(\mathcal{S}, c) ; Y^{\mathrm{AWGN}}(\overline{\mathcal{S}}, c) \mid X^{\mathrm{AWGN}}(\overline{\mathcal{S}}, c)\right)\right. \\
& \leq I\left(\left(X^{\mathrm{poly}}(\mathcal{S}, c) ; Y^{\mathrm{poly}}(\overline{\mathcal{S}}, c) \mid X^{\text {poly }}(\overline{\mathcal{S}}, c)\right)+9 d_{\max } \log d_{\max }\right.
\end{aligned}
$$

for any of the $\mathrm{BC}$ and MAC components $c$. Plugging into (19) and noting that there are at most $c_{\max }$ components in any cut yields step (c) of the proof.

Steps (g) and (a) on the DSM are similar to the corresponding steps (e) and (c) in the AWGN model. In [7], we showed that

$$
\sum_{i \in \mathcal{S}} R_{i, \mathrm{BC}}^{\mathrm{DSM}} \geq \log \left(\max _{i \in \mathcal{S}}\left|h_{i}\right|^{2}\right)-7|\mathcal{S}| .
$$

is achievable on the $\mathrm{BC}$ in the DSM. For the MAC in the DSM we have

$$
\begin{aligned}
\sum_{i \in \mathcal{S}} R_{i, \text { MAC }}^{\text {DSM }} & \geq \log \left(\max _{i \in \mathcal{S}}\left|h_{i}\right|^{2}\right)-2 \log ((|\mathcal{S}|-1) !)-4|\mathcal{S}|-2 \\
& \geq \log \left(\max _{i \in \mathcal{S}}\left|h_{i}\right|^{2}\right)-6|\mathcal{S}| \log |\mathcal{S}|,
\end{aligned}
$$

where the first inequality is due to our result in [8]. Hence, we can achieve the polynomial rates $\rho^{\text {in }}$ and $\rho^{\text {out }}$ on the BC and MAC components, which yields step (a), $\mathcal{R}_{\text {ach }}^{\mathrm{DSM}} \supseteq \mathcal{R}_{\text {ach }}^{\text {poly }}$, by using decode-and-forward in the multiple-unicast network.

Finally, a cut in the DSM network separates as (19). Due to [8], the sum-rate achieved by a subset $\mathcal{S}$ of cooperating transmitters in the DSM MAC is upper bounded as

$$
\begin{aligned}
\sum_{i \in \mathcal{S}} R_{i, \text { MAC }}^{\text {DSM }} & \leq 2 \log \left(\sum_{i \in \mathcal{S}}\left|h_{i}\right|\right)+1 \\
& \leq 2 \log \left(|\mathcal{S}| \max _{i \in \mathcal{S}}\left|h_{i}\right|\right)+1 \\
& \leq \log \left(\max _{i \in \mathcal{S}}\left|h_{i}\right|^{2}\right)+2|\mathcal{S}| \log |\mathcal{S}| \\
& \leq \rho^{\text {in }}+9 d_{\max } \log d_{\text {max }} .
\end{aligned}
$$

The sum-rate of the DSM BC with cooperating receivers is [7]

$$
\begin{aligned}
\sum_{i \in \mathcal{S}} R_{i, \mathrm{BC}}^{\mathrm{DSM}} & \leq \log \left(\max _{i \in \mathcal{S}}\left|h_{i}\right|^{2}\right)+|\mathcal{S}| \log 6 \\
& \leq \log \left(\max _{i \in \mathcal{S}}\left|h_{i}\right|^{2}\right)+3|\mathcal{S}| \log |\mathcal{S}| \\
& \leq \rho^{\text {out }}+10 d_{\max } \log d_{\text {max }} .
\end{aligned}
$$


With (19), the value of a cut $\mathcal{S}$ in the DSM multiple-unicast network is bounded as

$$
\begin{aligned}
I( & \left(X^{\mathrm{DSM}}(\mathcal{S}) ; Y^{\mathrm{DSM}}(\overline{\mathcal{S}}) \mid X^{\mathrm{DSM}}(\overline{\mathcal{S}})\right) \\
\leq & \sum_{c} I\left(\left(X^{\mathrm{DSM}}(\mathcal{S}, c) ; Y^{\operatorname{DSM}}(\overline{\mathcal{S}}, c) \mid X^{\mathrm{DSM}}(\overline{\mathcal{S}}, c)\right)\right. \\
\leq & \sum_{c}\left(I \left(\left(X^{\text {poly }}(\mathcal{S}, c) ; Y^{\mathrm{poly}}(\overline{\mathcal{S}}, c) \mid X^{\text {poly }}(\overline{\mathcal{S}}, c)\right)\right.\right. \\
& \left.\quad+10 d_{\max } \log d_{\text {max }}\right) \\
= & I\left(\left(X^{\text {poly }}(\mathcal{S}) ; Y^{\text {poly }}(\overline{\mathcal{S}}) \mid X^{\text {poly }}(\overline{\mathcal{S}})\right)\right. \\
& +c_{\max } 10 d_{\max } \log d_{\max },
\end{aligned}
$$

where (27b) is due to (25) and (26), and (27c) is due to the separation of the cut-set bound in the polymatroidal network [5]. This yields step (g), $\mathcal{R}_{\text {cut }}^{\text {DSM }} \subseteq \mathcal{R}_{\text {cut }}^{\text {poly }}+\alpha$, and concludes the proof of Theorem 2 .

\section{DISCUSSION AND CONCLUSION}

Our result shows that the capacity region of AWGN multiple-unicast networks can be approximated by the capacity region of the corresponding network in the DSM. Previously, this result was only known for single-unicast networks and for $K$-user interference channels.

Unfortunately, there is not only an additive gap $\alpha$ between the two regions, but also a multiplicative gap $\mu$. While the additive gap can be made relatively small (by increasing the SNR and, thus, the capacity regions), this is not possible for the multiplicative gap. The multiplicative gap results from Theorem 1, which connects the min-cut region and the maxflow region of the polymatroidal network. There is some indication that this multiplicative gap cannot be avoided for multiple-unicast: For multiple-unicast in wire-line graphs, the rate region achieved by routing and the min-cut region are within a multiplicative gap of $\mathcal{O}(\log k)$. Routing, however, is conjectured to be optimal, which would mean that the min-cut region is loose [5].

\section{REFERENCES}

[1] A. El Gamal and Y.-H. Kim, Network Information Theory. Cambridge University Press, 2011.

[2] A. Avestimehr, S. Diggavi, and D. Tse, "Wireless network information flow: A deterministic approach," IEEE Trans. Inf. Theory, vol. 57, no. 4, pp. 1872 - 1905, Apr. 2011.

[3] R. Etkin, D. Tse, and H. Wang, "Gaussian interference channel capacity to within one bit," IEEE Trans. Inf. Theory, vol. 54, no. 12, pp. 55345562, Dec. 2008

[4] M. Anand and P. Kumar, "A digital interface for Gaussian relay and interference networks: Lifting codes from the discrete superposition model," IEEE Trans. Inf. Theory, vol. 57, pp. 2548-2564, May 2011.

[5] S. Kannan and P. Viswanath, "Capacity of multiple unicast in wireless networks: A polymatroidal approach," arXiv:1111.4768v1, Nov. 2011.

[6] Ford, L.R., Jr. and D. Fulkerson, "Maximal flow through a network," Canad. J. Math., vol. 8, pp. 399-404, 1956.

[7] N. Schrammar, M. Andersson, and M. Skoglund, "Approximate capacity of the general Gaussian parallel relay network," in Proc. Int. Symp. Inf. Theory (ISIT), 2011.

[8] N. Schrammar and M. Skoglund, "Capacity bounds for the discrete superposition model of the Gaussian multiple-access channel," in Proc. IEEE Wirel. Comm. \& Netw. Conf. (WCNC), Mar. 2011. 